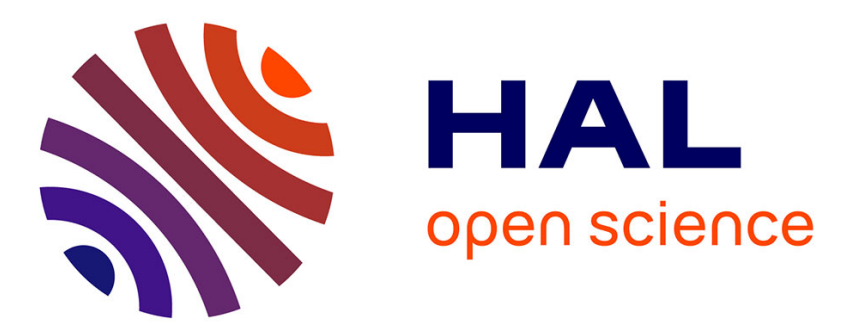

\title{
Phénomènes d'interférences dépendant du flux lumineux dans les processus résonnants d'ionisation multiphotonique
}

\author{
S. Feneuille, L. Armstrong
}

\section{- To cite this version:}

S. Feneuille, L. Armstrong. Phénomènes d'interférences dépendant du flux lumineux dans les processus résonnants d'ionisation multiphotonique. Journal de Physique Lettres, 1975, 36 (10), pp.235-237. 10.1051/jphyslet:019750036010023500 。 jpa-00231196

\section{HAL Id: jpa-00231196 https://hal.science/jpa-00231196}

Submitted on 1 Jan 1975

HAL is a multi-disciplinary open access archive for the deposit and dissemination of scientific research documents, whether they are published or not. The documents may come from teaching and research institutions in France or abroad, or from public or private research centers.
L'archive ouverte pluridisciplinaire HAL, est destinée au dépôt et à la diffusion de documents scientifiques de niveau recherche, publiés ou non, émanant des établissements d'enseignement et de recherche français ou étrangers, des laboratoires publics ou privés. 


\title{
PHÉNOMÈNES D'INTERFÉRENCES DÉPENDANT DU FLUX LUMINEUX DANS LES PROCESSUS RÉSONNANTS D'IONISATION MULTIPHOTONIQUE
}

\author{
S. FENEUILLE \\ Laboratoire Aimé-Cotton, C.N.R.S. II, Bât. 505, 91405 Orsay, France \\ L. ARMSTRONG Jr. $\left({ }^{*}\right)$ \\ Department of Physics, The Johns Hopkins University Baltimore, \\ Maryland 21218, U.S.A.
}

(Reçu le 5 juin 1975, accepté le 9 juillet 1975)

\begin{abstract}
Résumé. - L'objet de cette lettre est de montrer que dans certains cas limites physiquement réalisables, on peut donner une expression analytique du profil d'une section efficace d'ionisation multiphotonique au voisinage d'une résonance. Dans ces conditions, il est possible de suivre, avec l'intensité du flux lumineux incident, l'évolution des phénomènes observés (élargissement et déplacement) et ainsi de mieux comprendre l'origine de ces derniers.
\end{abstract}

Abstract. - The object of this letter is to show, that, in some limiting cases, one can give an analytical expression of the profile of multiphoton ionization cross-sections near a resonance. Under these conditions, the light flux dependence of the phenomena becomes very clear and the origin of the shift and the broadening of the resonance can be easily understood.

L'ionisation multiphotonique est généralement décrite dans le formalisme de la théorie des perturbations dépendant du temps et, pour un processus donné, seul est retenu, en première approximation, le terme non nul d'ordre le plus bas qui apparaît dans le développement de l'opérateur S [1]. Naturellement, cette approximation cesse d'être valable si l'on travaille avec des flux lumineux suffisamment élevés et, dans ce cas, les effets d'ordre supérieur peuvent jouer un rôle important, en particulier au voisinage d'une résonance, comme le montrent un certain nombre de calculs récents [2] dont certaines des prédictions ont d'ailleurs été vérifiées expérimentalement [3]. Cependant, le fait de prendre en compte, dans le développement de l'opérateur $\mathrm{S}$, des termes d'ordre de plus en plus élevé conduit à des calculs fort complexes qui, en général, ne peuvent être conduits que de façon numérique; il est donc difficile d'en extraire des conclusions générales et, par là même, une interprétation physique simple des nouveaux effets observés.

L'objet de cette lettre est de montrer qu'en utilisant le modèle de l'atome habillé [4], on peut donner une

$\left(^{*}\right)$ Work supported in part by The National Science Foundation. expression analytique du profil de la section efficace au voisinage d'une résonance dans un cas limite correspondant à une situation physique effectivement rencontrée. Dans ces conditions, il est alors possible de suivre, avec l'intensité du flux lumineux incident, l'évolution des phénomènes observés et ainsi de mieux comprendre l'origine de ces derniers.

$\mathrm{Au}$ voisinage d'une résonance, nous décrivons le phénomène d'ionisation multiphotonique de la façon suivante :

i) un processus direct qui, grâce à la présence d'autres états intermédiaires, apparaîtrait même en l'absence de l'état quasi résonnant $\varphi$; ce processus direct à $n$ photons est induit par une interaction , effective $H_{n}^{E}$ entre l'atome et le champ ;

ii) un processus à deux étages qui se décompose en une transition à $p$ photons de l'état initial $i$ à l'état quasi résonnant $\varphi$ (cette première excitation étant induite par une interaction effective $H_{P}$ ), suivie d'une transition à $(n-p)$ photons de l'état $\varphi$ vers un état du continuum $\Psi_{E}$ (cette seconde excitation étant induite par l'interaction effective $H_{n-p}^{E}$ ).

Pour des raisons de simplicité, tous les états considérés sont supposés non dégénérés et l'on néglige les phénomènes d'émission spontanée.

Dans le modèle de l'atome habillé, l'action du 
champ électromagnétique, qui est décrit par $m$ photons de fréquence $\omega / 2 \pi$ et de polarisation données, est, d'une part, de rendre quasi dégénérés les états $|i, m\rangle$, $|\varphi, m-p\rangle$ et $\left|\Psi_{E}, m-n\right\rangle$, d'autre part, de mélanger ces états par l'intermédiaire de l'interaction effective :

$$
\begin{aligned}
& H_{\mathrm{INT}}=\left\{\int|i, m\rangle H_{n}^{E^{\prime}}\left\langle\Psi_{E^{\prime}}, m-n\right| \mathrm{d} E^{\prime}+\right. \\
& +|i, m\rangle H_{P}\langle\varphi, m-p| \\
& \left.+\int|\varphi, m-p\rangle H_{n-p}^{E^{\prime}}\left\langle\Psi_{E^{\prime}}, m-n\right| \mathrm{d} E^{\prime}\right\}+ \text { C.C. }
\end{aligned}
$$

La prise en compte du mélange simultané de ces états ne conduit pas à un système analytiquement soluble. Cependant, il est clair que l'on peut négliger le mélange des états du continuum avec l'état initial puisqu'il n'intervient pas directement dans le processus résonnant. En outre, si l'on suppose

$$
\begin{aligned}
\left|\left\langle i, m\left|H_{\mathrm{INT}}\right| \varphi, m-p\right\rangle\right| \gg \\
\quad \gg\left|\left\langle\varphi, m-p\left|H_{\mathrm{INT}}\right| \Psi_{E}, m-n\right\rangle\right|^{2},
\end{aligned}
$$

on peut également négliger le mélange des états du continuum avec l'état quasi résonnant, et seul reste à considérer dans ce cas le mélange entre l'état fondamental et l'état quasi résonnant. La condition précédente sera en particulier satisfaite dans tout processus résonnant d'ionisation à deux photons; de façon plus générale, la validité de cette approximation ne sera assurée que pour $p \ll n-p$.

Le fait de prendre en compte ce dernier mélange revient à substituer aux états non perturbés $|i, m\rangle$ et $|\varphi, m-p\rangle$, l'état $\Phi(t)$ suivant qui décrit le phénomène bien connu de nutation :

$$
\begin{aligned}
\Phi(t)=\cos \theta \exp \left(\check{-} i \lambda_{+}\left(t-t_{0}\right)\right) \Phi_{+}+ \\
+\sin \theta \exp \left(-i \lambda_{-}\left(t-t_{0}\right)\right) \Phi_{-},
\end{aligned}
$$

où $t_{0}$ est un temps arbitraire,

$$
\begin{gathered}
\Phi_{+}=\cos \theta|i, m\rangle-\sin \theta|\varphi, m-p\rangle \\
\Phi_{-}=\sin \theta|i, m\rangle+\cos \theta|\varphi, m-p\rangle \\
\cos \theta=\left\{\frac{W+\delta}{2 W}\right\}^{1 / 2}, \sin \theta=\left\{\frac{W-\delta}{2 W}\right\}^{1 / 2}, \\
W=\left[K^{2}+\delta^{2}\right]^{1 / 2}, \delta=\frac{1}{2}\left[\Omega_{\varphi}-\Omega_{i}-p \omega\right], \\
K=\hbar^{-1}\left\langle i, m\left|H_{\mathrm{INT}}\right| \varphi, m-p\right\rangle, \\
\lambda_{ \pm}=\Omega \mp W, \\
\Omega=\Omega_{i}+m \omega+\delta=\Omega_{\varphi}+(m-p) \omega-\delta,
\end{gathered}
$$

$\hbar \Omega_{i}$ et $\hbar \Omega_{\varphi}$ étant les énergies non perturbées respectives des états $i$ et $\varphi$; sans perte de généralité, $K$ est supposé réel.
Dans ces conditions la probabilité totale d'exciter l'atome dans l'état $\Psi_{E}$ est proportionnelle à :

$$
\begin{aligned}
\cos ^{2} \theta \delta(E+(m & \left.-n) \hbar \omega-\hbar \lambda_{+}\right) \times \\
& \times\left|\left\langle\Phi_{+}\left|H_{\mathrm{INT}}\right| \Psi_{E}, m-n\right\rangle\right|^{2} \\
& +\sin ^{2} \theta \delta\left(E+(m-n) \hbar \omega-\hbar \lambda_{-}\right) \\
& \times\left|\left\langle\Phi_{-}\left|H_{\mathrm{INT}}\right| \Psi_{E}, m-n\right\rangle\right|^{2}
\end{aligned}
$$

Si les éléments de matrice

$$
\begin{aligned}
& J_{E}=\left\langle i, m\left|H_{\mathrm{INT}}\right| \Psi_{E}, m-n\right\rangle \\
& L_{E}=\left\langle\varphi, m-p\left|H_{\mathrm{INT}}\right| \Psi_{E}, m-n\right\rangle,
\end{aligned}
$$

que l'on peut d'ailleurs supposer réels, varient lentement avec $E$, on obtient en négligeant cette variation :

$P_{\mathrm{T}} \propto \frac{W^{2}+\delta^{2}}{2 W^{2}} J^{2}+\frac{W^{2}-\delta^{2}}{2 W^{2}} L^{2}-\frac{\delta}{W^{2}}\left(W^{2}-\delta^{2}\right)^{1 / 2} J L$

le premier terme décrit le processus direct, le second, le processus à deux étages et le troisième, l'interférence entre ces deux processus. Si $P_{\mathrm{D}}$ est la probabilité d'excitation relative au seul processus direct, on peut finalement écrire :

$$
\frac{P_{\mathrm{T}}}{P_{\mathrm{D}}}=\frac{1}{2}+\frac{(\delta-K a)^{2}}{2\left(\delta^{2}+K^{2}\right)}
$$

où $a=L / J$.

On obtient ainsi un profil asymétrique présentant un minimum pour $\delta_{m}=K a$ et un maximum pour $\delta_{M}=-K / a$, cette asymétrie étant due uniquement au terme d'interférence. Pour préciser l'évolution de ce profil avec le flux incident, il suffit de remarquer que $K, J$ et $L$ peuvent être réécrits :

$$
\begin{aligned}
& K=[m ! /(m-p) !]^{1 / 2} k \simeq m^{p / 2} k, \\
& J=[m ! /(m-n) !]^{1 / 2} j \simeq m^{n / 2} j, \\
& L=[(m-p) ! /(m-n) !]^{1 / 2} l \simeq m^{(n-p) / 2} l,
\end{aligned}
$$

$k, j$ et $l$ étant des quantités indépendantes du flux incident. On constate que la position du minimum est indépendante du flux incident, alors que celle du maximum, $\delta_{M}=m^{p} k j / l$, en dépend essentiellement. Ce déplacement de la résonance peut être interprété comme un effet Stark dynamique [5]. Bien entendu, lorsque le flux lumineux augmente, la résonance est non seulement déplacée mais également élargie (voir Fig. 1).

Une autre façon d'apprécier l'importance des effets d'ordre supérieur est de considérer l'ordre effectif de non-linéarité défini par $\mathrm{d}(\log \sigma) / \mathrm{d}(\log m)$ où $\sigma$ est la section efficace totale de photoionisation. Les résultats précédents conduisent à :

$$
\begin{aligned}
& \frac{\mathrm{d}(\log \sigma)}{\mathrm{d}(\log m)}=(n-1)-p A^{2} \times \\
& \times \frac{(y-1)^{2}}{\left(A^{2}+y^{2}\right)\left(A^{2}+y^{2}+[y-1]^{2}\right)}
\end{aligned}
$$




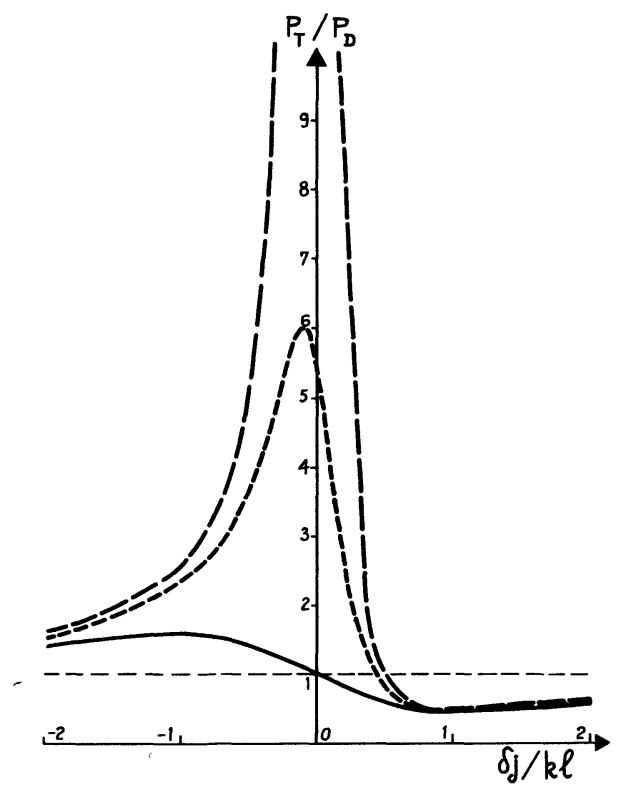

FIG. 1. - Influence du mélange de l'état initial et de l'état quasi résonnant sur la section efficace d'ionisation multiphotonıque pour différentes valeurs du flux lumineux incident

$\left(A^{2}=m^{p} j^{2} / l^{2}\right):---A^{2} \rightarrow 0 ;--A^{2}=0,1 ;-A^{2}=1$.

où $A^{2}=m^{p} j^{2} / l^{2}$ et $y=\delta j / k l$; ceci montre que dans le cas particulier considéré $(p \ll n-p)$, l'ordre effectif de non-linéarité reste toujours inférieur à $(n-1)$ sauf quand $\delta=k l / j$, c'est-à-dire quand la section efficace est à son minimum (voir Fig. 2). Naturellement, lorsqu'on s'éloigne de la résonance, on retrouve une valeur très proche de $(n-1)$. En outre, comme cela aurait pu être facilement prévu, pour de faibles valeurs de $A^{2}$, l'ordre effectif de nonlinéarité reste très voisin de $(n-1)$ quel que soit $\delta$, différent de zéro.

Ainsi, le modèle de l'atome habillé permet d'obtenir, dans un cas limite qui peut être physiquement réalisé, le profil d'une section efficace d'ionisation multiphotonique au voisinage d'une résonance sous forme d'une fonction analytique très simple de l'écart à la résonance, dépendant de deux paramètres. Ces derniers peuvent être déterminés à partir de l'intensité du flux lumineux incident et des seules grandeurs

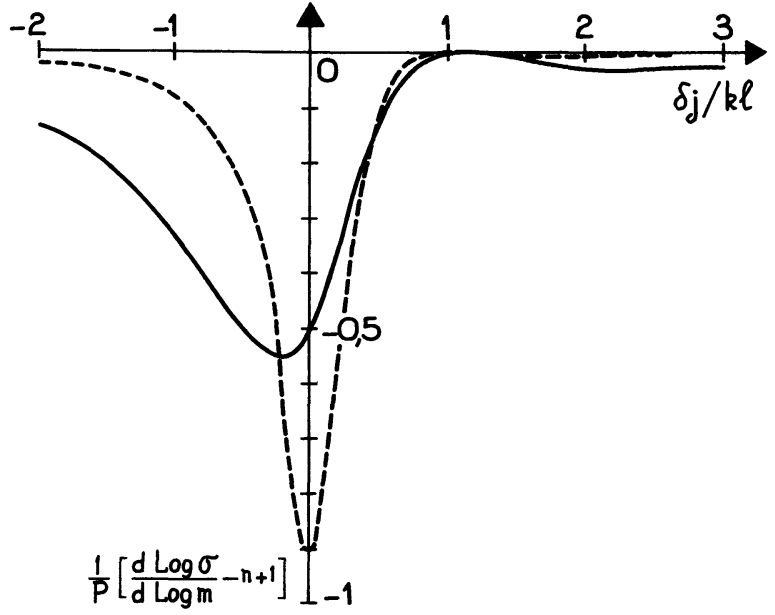

Fig. 2. - Influence du mélange de l'état initial et de l'état quasi résonnant sur l'ordre effectif de non-linéarité d'un processus résonnant d'ionisation multiphotonique ( $\left.\ldots-A^{2}=0,1 ;-A^{2}=1\right)$.

atomiques qui interviennent dans le calcul du terme non nul d'ordre le plus bas apparaissant dans le développement de l'opérateur S. Par ailleurs, ces paramètres pourraient être considérés comme des grandeurs ajustables aux données expérimentales, ce qui permettrait de vérifier de façon immédiate la validité des approximations choisies.

Il est à noter qu'un autre cas limite peut être également traité par la méthode décrite ici : celui où le mélange entre l'état fondamental et l'état quasi résonnant est négligeable devant le mélange de ce même état quasi résonnant avec les états du continuum (cas où $p \gg n-p$ ). La façon la plus simple de tenir compte de ce dernier est de tirer parti de l'analogie profonde entre le problème considéré et celui de l'autoionisation. Le formalisme introduit par Fano [6] peut être en fait immédiatement transposé et l'on obtient à nouveau, pour la section efficace au voisinage d'une résonance, un profil asymétrique qui dépend du flux lumineux mais de façon plus complexe que dans le cas étudié dans cette lettre. Les résultats détaillés correspondants seront publiés ultérieurement [7].

\section{Bibliographie}

[1] Voir par exemple : Gontier, Y. et Trahin, M., Phys. Rev. A 4 (1971) 1896.

[2] Zon, B. A., Manakov, N. L. et Rapoport L. P., Sov. Phys. JETP 34 (1973) 515.

Gontier, Y. et Trahin, M., Phys. Rev. A7 (1973) 2069.

Chang, C. S. et Stehle, P., Phys. Rev. Lett. 30 (1973) 1283.

Lambropoulos, P., Phys. Rev. A 9 (1974) 1992.

Gersten, L. et Mitrleman, M. H., Phys. Rev. A 10 (1974) 74.

Geltman, S. et Teague, M. R., J. Phys. B 7 (1974) L22.

[3] Agostini, P., Barjot, C., Mainfray, G., Manus, C. et ThéBaUlt, J., Phys. Lett. 31A (1970) 367.
Bacos, J., Kiss, A., Szabó, L. et Tendler, M., Phys. Lett. 41A (1972) 163.

Held, B., Mainfray, G., Manus, C., Morellec, J. et Sanchez, F., Phys. Rev. Lett. 30 (1973) 423.

[4] Cohen-Tannoudj, C. et Haroche, S., J. Physique 30 (1969) 125.

[5] Autler, S. H. et Townes, C. H., Phys. Rev. 100 (1955) 703.

[6] Fano, U., Phys. Rev. 124 (1961) 1866.

[7] Armstrong, L., Jr., Beers, L. B. et Feneuille. S.. à naraitre dans Phys. Rev. 